\title{
Key physicochemical properties of nanomaterials in view of their toxicity: an exploratory systematic investigation for the example of carbon-based nanomaterial
}

\author{
Beatrice Salieri • Andrea Pasteris • \\ Woranan Netkueakul • Roland Hischier
}

Received: 7 July 2016 / Accepted: 20 January 2017

(C) Springer Science+Business Media Dordrecht 2017
SWCNT, MWCNT, $\mathrm{C}_{60}$-fullerene, functionalization), while multiple regression analysis was used to assess the effect of quantitative properties (i.e., diameter length of nanotubes, secondary size) on the toxicity values. The here described investigations revealed significant relationships among the physicochemical properties and observed toxic effects. The research was mainly affected by the low availability of data and also by the low variability of the studies collected. Overall, our results demonstrate that the here proposed and applied approach could have a major role in identifying the physicochemical properties of relevance for the toxicity of nanomaterial. However, the future success of the approach would require that the ENMs and the experimental conditions used in the toxicity studies are fully characterized.

Keywords Carbon-based nanomaterial $\cdot$ Ecotoxicity Physicochemical properties $\cdot$ Exploratory investigation . ANOVA $\cdot$ Regression analysis

\section{Introduction} article (doi:10.1007/s11051-017-3748-3) contains supplementary material, which is available to authorized users.

B. Salieri $(\bowtie) \cdot$ W. Netkueakul $\cdot$ R. Hischier Technology and Society Lab, Empa, Swiss Federal Laboratories for Materials Science and Technology, Lerchenfeldstrasse 5, CH9014 St. Gallen, Switzerland e-mail: Beatrice.salieri@empa.ch

A. Pasteris

Department of Biological, Geological, and Environmental Sciences, University of Bologna, via Sant'Alberto 163, 48121 Ravenna, Italy
Engineered nanomaterials (ENMs) contribute nowadays more and more to technological, social, and economic innovation; however, scientific concerns on, e.g., potential human and environmental impacts have been raised (Subramanian et al. 2015; Valverde and Linkov 2011; Aschberger et al. 2011a, b). One issue is that the novel properties of ENMs may increase their toxicological potential compared to the classical (microscale) form of the same substance (Rossi et al. 2014). Although studies have 
been conducted to assess the potential risk of these emerging contaminants (Coll et al. 2016; Gottschalk et al. 2013; Mueller and Nowack 2008; Gottschalk et al. 2009), a full characterization of the potential human health and ecotoxicological impacts has yet to be achieved (Aschberger et al. 2011a, b; Handy et al. 2008). The influence of the physicochemical properties of ENMs on their toxicity has thus far not been clarified (Liu et al. 2013), while large variabilities in observed effects, even within the same test species, are reported in literature (Handy et al. 2012). The current situation is mainly the result of (i) the scarcity of full characterization studies of ENMs in test and environmental media, and (ii) the use of nonstandardized tests and test materials (Guarch et al. 2014; Oomen et al. 2014; RiveraGP et al. 2012).

Hence, we currently have a noncomprehensive understanding of the mechanism of toxicity of ENMs and of their potential hazard and environmental behavior. In this context, the understanding of the physicochemical properties driving toxicity is of high relevance. As opposed to, for example, an organic chemical such as benzene, there is not one single form of the nanomaterial xy but numerous "variations" with differences in size, form, and surface coating, while respective differences in the overall physicochemical properties can be found for each ENM (Park et al. 2015).

Thus, a grouping approach of ENMs based on their intrinsic material properties, system-dependent properties, specific type of exposure route, uptake, bio-distribution, and toxic effect seems to be a next step in order to be able to characterize these substances in a more consistent way (Arts et al. 2015). The European Chemical Agency (ECHA) describes "grouping" as a process of unifying into a common group all substances that are likely to have similar physical, chemical, toxicological, ecotoxicological, and/or environmental fate properties or that follow a regular pattern (ECHA 2013). Thanks to the development of a grouping approach, the biological activity of a substance, belonging to a specific group, instead of being directly measured, may be derived from the activities measured for other substances within the same group. Furthermore, models for predicting the toxicity of ENMs, based on their psychochemical properties, may be developed as an alternative to standard bioassay (Arts et al. 2015).

Such a grouping based on the physicochemical properties of ENM would also be very valuable in the field of life cycle assessment (LCA). According to the ISO 14' 040 standard, LCA is a tool to estimate and assess the environmental performance of a material or product, i.e., their potential environmental impacts, as related to a specific question (ISO 2006). Traditionally, massbased inputs and outputs flows in LCA modeling (e.g., input of chemical substances, releases into the environment, etc.) are expressed as an amount and measured in kilograms. The common life cycle impact assessment (LCIA) approach for the assessment of toxicity, the USEtox $^{\mathrm{TM}}$ model, (Rosenbaum et al. 2008) is therefore applying mass-based characterization factors (CFs) for the assessment of the ecotoxicological and human toxicological impacts of releases. However, mass might not be the unit of measurement that is most relevant when considering potential impacts of ENP releases to the environment and, consequently, might not be the most suitable unit in LCIA, as highlighted by various researchers (Hischier 2014; Hischier and Walser 2012). Rather, other material properties, such as surface area or number concentration, might be more appropriate measurement units. Hence, a characterization of ENMs based on their physicochemical properties could be valuable as a precursor to the definition of appropriate CFs for ENM releases, while a grouping within these properties would allow for the reducing in the number of different CFs that may be required for each ENM.

This paper presents a first attempt to identify the key physicochemical properties that affect the toxicity of ENMs. For this, we investigated the relationship between the physicochemical properties of ENMs and their toxic values by means of statistical techniques, including analysis of variance (ANOVA) and regression analysis. Those two statistical techniques enable (1) to analyze of whether there are any statistically significant differences among group means and (2) the identification of the type of relationship among variables. ANOVA and the regression analysis have been here applied due to their well-known robustness. The ANOVA technique is commonly used to define the statistical significance between qualitative (categorical) properties (e.g., exact type of a nanomaterial) and quantitative properties (e.g., $\mathrm{EC}_{50}$ ). Here, ANOVA was used to determine the significance of the effects of the qualitative properties on the toxicity of the examined nanomaterials. This analysis was then extended by using regression models, allowing for the description of the relationship between properties and the toxicity based on a regression coefficient $(\beta)$.

All these investigations have been performed here on carbon-based nanomaterials (CBNs), which are a class of ENMs that comprise carbon nanotubes (i.e., multiwall 
carbon nanotubes, MWCNT, and single-wall carbon nanotubes, SWCNT), spherical fullerene $\left(\mathrm{C}_{60}\right)$, graphene, and mesoporous carbon structures. CBNs have considerable potential for application in a number of industrial and consumer sectors, and due to their exceptional mechanical, electronic, physical, and chemical properties, they will play a key role in the development of modern advanced materials, such as super-capacitors, transistors, and transparent photoelectronic devices or sensors. However, this growing use of CBNs has raised several concerns on their safety and environmental impact (Jackson et al. 2013; Subramanian et al. 2015). Indeed, previous studies have shown that the toxicity of CBNs to freshwater organisms is affected by several mechanisms of toxicity and physicochemical properties. For algal species, key mechanisms of toxicity include agglomeration and physical interaction of CBNs with cells, shading effect and induced oxidative stress (i.e., by metal impurities), internalization of carbon nanotubes that might induce membrane ruptures, and morphological alterations (Luo 2007; Gao et al. 2012; Youn et al. 2012; Long et al. 2012; Pereira et al. 2014; Sohn et al. 2015). For crustaceans species (Daphnia magna, Daphnia pluex, Ceriodophnia dubia), the ingestion of agglomerated forms, the adhesion of aggregated forms to the external surface, and the presence of CBNs that may interfere with the feeding mechanism have been suggested (Gao et al. 2012; Kapler et al. 2009; Arndt et al. 2013; Yan et al. 2010; Zhu et al. 2006; Luo 2007; Seda et al. 2012; Alloy and Roberts 2011; Edgington et al. 2010; Li and Huang 2011; Kim et al. 2010; Roberts et al. 2007). As well, oxidative stress and the decreasing of photoirradiation potential due to the agglomerated forms of CBNs have been identified as of relevance for in vivo toxicity to fish (Usenko et al. 2008). Testing procedures (e.g., exposure time, use of solvent, dispersion method) have also been shown to potentially affect toxicity. Schwab et al. (2011) found that a well dispersed MWCNT solution was more toxic to algae (Chlorella vulgaris) than a 3-day-old agglomerated suspension and fresh agglomerated MWCNT dispersion. Furthermore, sonication time, exposure time, and presence of natural organic matter (NOM) may also influence the toxicity test (Zhu et al. 2006; Tao et al. 2009; Gao et al. 2012; Pereira et al. 2014; Edgington et al. 2010; Cheng et al. 2007). Currently, the toxicity of CNT is determined by a set of physiochemical properties namely secondary size as agglomerated forms, hydrophobicity (Zhu et al. 2009), surface functionalization and impurities, i.e., hydroxyl group (Usenko et al. 2007; Arndt et al. 2013; Pereira et al. 2014; Cheng et al. 2007; Sohn et al. 2015), the length (Cheng et al. 2007), surface charge and area (Edgington et al. 2010; Long et al. 2012), and by system-dependent properties (e.g., pH), and thus, the hazard assessment of a single material type requires a property combination set (Oomen et al. 2014). Thus, several physicochemical properties (e.g., colloidal stability, purity, inertness, size, shape, charge) are potentially of relevance for the eco-toxicity of CBNs. To date, an experimental correlation of these properties with ecotoxicity is still under investigation. However, this is hard to perform since the various properties are strongly mutually connected (Rivera-GP et al. 2012), and it is therefore difficult to vary individual properties of ENMs while keeping the others constant. Therefore, a statistical investigation is proposed here as a first attempt to characterize the key properties of relevance based on the published ecotoxicity studies performed on freshwater algae, crustacean, and fish.

\section{Material and methods}

In a first step, an extensive literature survey concerning the ecotoxicity of MWCNT, SWCNT, and $\mathrm{C}_{60}$ fullerenes has been conducted. Half-maximal effect concentration $\left(\mathrm{EC}_{50}\right)$ values were collected from in vivo toxicity studies carried out on freshwater organism representative of the trophic levels of algae, crustaceans, and fish (Table 1). For the survey (conducted in March 2016), we searched for the query (toxicity OR $\mathrm{EC}_{50}$ ) AND (MWCNT OR SWCNT OR $\mathrm{C}_{60}$ ) AND (algae OR crustaceans OR fish) in the databases of ScienceDirect and Google Scholar. In total, 29 articles were identified (see Table 1). Toxic effects, expressed as lowest observed effect concentration (LOEC) and nonobserved effect concentration (NOEC), were, a priori, excluded to avoid conversion factors. Also recorded for each study were the physicochemical properties (e.g., length, primary diameter, surface area, etc.) and system-dependent properties (e.g., secondary diameter measured by TEM/DLS or Zeta potential, dispersant, etc.) of the examined substances, the tested organism, and the measured endpoint. The data collected are reported in Supporting Information (Table S1-S9).

In a second step, investigations of the relationship between those physicochemical properties and the toxic values were conducted by using statistical approaches. Specifically, ANOVA was used to assess the effect of categorical properties on the toxicity values of each ENM. Each property was tested separately, i.e., one 
separate test was performed for each property, which was taken as the single factor (categorical predictor) in a one-way ANOVA. The categorical properties assessed were use of a dispersant or treatments in the test medium (yes vs. no), type of ENM (SWCNT, MWCNT, $\mathrm{C}_{60}$ fullerene), and functionalization (yes vs. no). The $\log$ of $\mathrm{EC}_{50}$ was taken as the dependent variable. Only two levels were defined for the factors dispersant and functionalization, i.e., presence or absence. It was therefore not possible to consider each different dispersant or functionalization as a distinct level in the analysis as this would have caused a lack of replication and missing values.

Multiple regression analysis was used to assess the effect of quantitative properties (e.g., diameter length of nanotubes, secondary size) on the toxicity values. All the quantitative properties were tested simultaneously, using them as independent variables (predictors) in both a multiple linear regression model (Eq. (1)) and a multiple polynomial regression model (Eq. (2)):

$$
Y=\beta_{0}+\beta_{1} X_{1}+\beta_{2} X_{2}+\beta_{3} X_{3}+\ldots+\beta_{p} X_{p}+\varepsilon
$$

$Y=\beta_{0}+\beta_{1} X_{1}+\beta_{1} X_{1}^{2}+\beta_{2} X_{2}+\beta_{2} X_{2}^{2}+\ldots+\beta_{p} X_{p}^{2}+\varepsilon$

where $Y$ is the dependent variable, $X_{1}, X_{2}, \ldots X_{\mathrm{p}}$ are the independent variables (predictors), and $\varepsilon$ is the error term. The quantitative properties used as predictors were diameter, length (for nanotubes), secondary size, and duration of exposure. In addition, the use of a dispersant in the test medium (yes vs. no) was included as additional categorical independent variables in the regression models. The $\log _{10}$ EC50 was taken as the dependent variable. All the statistical analyses were carried out with the software tools MATLAB (functions: anova1, multicompare) and R (regression analysis, packages QuantPsyc).

\section{Results and discussion}

The numbers of articles identified through the literature survey are presented in Table 1. All collected data are listed in detail in the Supporting Information (Tables S1-S9).

An overall overview of the results obtained by the ANOVA and regression analysis for each one of the type of CBNs under investigation are reported in Table 2. Two aspects were excluded from the study due to a lack of available data: (1) toxicity effects of ENMs on fish and (2) the toxic effects of fullerene on each species.

Concerning the trophic level of algae, the type of CBNs under investigation (i.e., MWCNT or SWCNT) is not likely to be of relevance in terms of toxicity. The regression analysis (MRA) revealed the variables of length and dispersion as significant properties, while diameter and secondary size were found to be insignificant. In contrast, the aforementioned significant properties were found to be not significant predictors by PRA.

Concerning the trophic level of crustaceans, the ANOVA test revealed that toxicity is likely to be influenced by the type of CBNs tested (MWCNT, SWCNT, or fullerene). For the MWCNT (case of study length not included), MRA and PRA showed similar results, indicating that time and secondary size are significant and negative predictors. Notably, the quadratic coefficient of secondary size became positive and significant within the PRA analysis. These results indicate that a threshold, linked to the secondary size, may exist where, beyond which, the relationship with the toxic effect is reversed. However, a clear understanding of this trend is not yet obtainable due to the scarce number of observation collected here. For SWCNT, the regression analysis was not carried out due to the dearth of available data points (i.e., $\mathrm{EC}_{50}$ ). Because the lack of date regarding the physicochemical information was not possible to conduct the regression analysis (MRA and PRA) for $\mathrm{C}_{60}$-fullerene, it was, however, possible to perform the ANOVA test

Table 1 The number of collected articles (29 in total) containing toxicity data of CNMs on different testing organisms, which are divided into three different trophic levels

\begin{tabular}{|c|c|c|c|c|}
\hline Trophic level & Organisms & SWCNT & MWCNT & $\mathrm{C}_{60}$ \\
\hline Algae & $\begin{array}{l}\text { Pseudokirchneriella subcapitata, Chlorella vulgaris, } \\
\text { Chlorella sp., Chlamydomonas reinhardti }\end{array}$ & 3 & 3 & 2 \\
\hline Crustacean & Daphnia magna, Ceriodaphnia dubia & 6 & 8 & 7 \\
\hline Fish & $\begin{array}{l}\text { Carassius auratus, Danio rerio, Micropterus salmoides, } \\
\text { Oryzias latipes, Pimephales promelas }\end{array}$ & 1 & 1 & 7 \\
\hline
\end{tabular}


Table 2 Summary of the results obtained by the one-way ANOVA, and multiple linear regression analysis (MRA) and polynomial regression analysis (PRA) for SWCNT-MWCNT-fullerene on algae, crustacean, and fish

\begin{tabular}{|c|c|c|c|c|c|c|c|c|}
\hline & Method & Type of ENMs & Length & Diameter & $\begin{array}{l}\text { Secondary } \\
\text { size }\end{array}$ & Time & \begin{tabular}{|l|} 
Functionalisation \\
\end{tabular} & $\begin{array}{l}\text { Treatmentt/ } \\
\text { Dispersant }\end{array}$ \\
\hline \multirow{3}{*}{ Algae SWCNT - MWCNT } & ANOVA & WIIIIIII & & & & & & \\
\hline & MRA & & \multicolumn{2}{|c|}{ 亘 } & & & & \\
\hline & PRA & & \multicolumn{3}{|c|}{ UIIIIII } & & & DIIIIIIIS \\
\hline $\begin{array}{l}\text { Crustacean } \\
\text { MWCNT-SWCNT- } C_{60}\end{array}$ & ANOVA & & & & & & & \\
\hline \multirow{3}{*}{ Crustacean SWCNT } & ANOVA & & & & & & & \\
\hline & MRA & & & & & & & \\
\hline & PRA & & & & & & & \\
\hline Crustacean MWCNT & ANOVA & & & & & \multicolumn{2}{|r|}{ QIIIIIIIII } & \\
\hline \multirow{2}{*}{$\begin{array}{l}\text { Crustacean MWCNT } \\
\text { (Lenght included) }\end{array}$} & MRA & & N.A & & & & & \\
\hline & PRA & & N.A & & & & & \\
\hline \multirow{2}{*}{$\begin{array}{l}\text { Crustacean MWCNT } \\
\text { (Lenght not included) }\end{array}$} & MRA & & & & & & & \multirow{3}{*}{ WIIIIIII } \\
\hline & PRA & & & & & & & \\
\hline Crustacean $\mathrm{C}_{60}$ & ANOVA & & & & & & & \\
\hline
\end{tabular}

Black: significant $(p<0.05)$, Diagonal pattern: not significant $(p \geq 0.05)$; Horizontal line pattern: positive and significant $(p \leq 0.05)$; Vertical line pattern: negative and significant $(p \leq 0.05)$; Light Grey: property not included in the investigation

$N . A$ the coefficient is not estimable (i.e. due to collinearity or due to a low number of observation)

*The quadratic coefficient is positive and significant

based on the use of dispersant, which was found to have a significant effect.

Trophic level of algae

Identified ecotoxicity data for the trophic level of algae and the physiochemical properties for MWCNT, SWCNT, and fullerene are reported in Tables S1-S7.

\section{ANOVA}

The ANOVA analysis has been firstly carried out by grouping the $\mathrm{EC}_{50}$ values by the type of CBNs; two groups have been identified as SWCNT and MWCNT. For $\mathrm{C}_{60}$ fullerene, only two observations (i.e., $\mathrm{EC}_{50} \mathrm{~s}$ ) were available and, thus, it has not been included in the analysis. The difference between the mean $\log$ of $\mathrm{EC}_{50}$ of the two groups of nanotubes was not significant $(p=0.19)$. The ANOVA test was then carried out by grouping the $\mathrm{EC}_{50}$ values by the use of dispersant agent - as yes/no (as dispersant gum arabic (GA), bovine serum albumin (BSA), sodium dodecyl sulfate (SDS), and natural organic matter (NOM) were used in the toxicity tests). The effect of the use of a dispersant has been assessed as not significant $(p=0.12)$ on the toxicity of SWCNT and MWCNT on algae. The use of dispersant agents in toxicity tests is largely debated. BSA, NOM, and GA are natural materials used as dispersants in laboratory to produce a homogenous and stable dispersion of ENM. SDS is a synthetic material applied for the same purpose. Several limits and advantages have been already remarked by the use of natural or synthetic dispersant, as the use of dispersing agent might potentially change the behavior of ENM (Handy et al. 2012). The adverse biological effects of dispersant agents have been reported in the literature, such as damage to cell membranes or oxidative stress. Gao et al. (2012) reported a lower toxicity of GA-SWCNT on Raphidocelis subcapitata compared to SDS-SWCNT, while a different growth pattern of $R$. subcapitata in culture media of either SDS-SWCNT or GA-SWCNTs was also observed. An in vitro study has revealed that BSA has the potential to increase the dispersibility of pristine SWCNTs in aqueous 
media and showed that the use of BSA has the potential to enhance the internalization of pristine SWCNT even by nonphagocytic cells (Muzi et al. 2016). This evidence suggests that use and type of dispersant used in aqueous solutions should be considered with care. However, the outcome of the ANOVA test carried out here is in agreement with the observations of Sohn et al. (2015), who showed that the use of a dispersant agent (i.e., BSA was used for the preparation of the SWCNT suspensions) did not induce any difference in ecotoxicity tests of SWCNT on $R$. subcapitata and C. vulgaris compared to distillated water. To our knowledge, no other studies have compared the type of dispersant agent used with the distilled water. Hence, the outcomes of the ANOVA test are partially in agreement with literature findings. A further test has been conducted by comparing two groups of $\mathrm{EC}_{50}$ identified by the use of dispersant agents BSA and NOM, with a significant difference between the means of the two groups observed $(p=0.04)$. Thus, the type of dispersant applied may affect the toxicity results, with the $\mathrm{EC}_{50}$ values belonging to the NOM group are on average lower than those belonging to the BSA group.

\section{Regression analysis}

The regression analysis was performed by accounting the variables of diameter, length, secondary size, and by the use of dispersants as yes (i.e., GA, SDS, NOM) or no for SWCNT and MWCNT. As above, the data concerning the fullerene are not here used due to the scarcity of observational data. The multiple linear regression analysis is significant $(p \leq 0.05)$ and a good fit of the data is observable with $R^{2}=0.98$ (Fig. 1).
The linear $\beta$ coefficients are reported in Table 3. Only the variables of length and dispersant are assessed as significant predictors, with the diameter and the secondary size found to be not significant predictors.

The influence of the dependent variables on the independent variable is accounted by comparing the magnitude of standardized $\beta$ coefficients of each property. This reveals that the length and the solvent are the most influent property (Fig. 2).

The polynomial regression analysis was not significant ( $\left.p=0.06 ; R^{2}=0.90\right)$. In summary, the regression analysis suggests that the use of a dispersant increases the toxicity of SWCNTs and MWCNTs on algae, that longer nanotubes are less toxic to these organisms, and that the relationship between length and $\log \mathrm{EC}_{50}$ is linear. The significant effect of the use of a dispersant is in contrast with the result of the one-way ANOVA. A possible explanation is that the multiple regression took separately into account the effect of each variable included in the model, whereas in the one-way ANOVA, the effect of the quantitative predictors was not explicitly taken into account, thereby inflating the within-groups variance and masking the effect of the dispersant.

\section{Crustaceans}

The physicochemical information together with the ecotoxicity data for the trophic level of crustaceans for MWCNT, SWCNT, and $\mathrm{C}_{60}$-fullerene is reported in Tables S2-S8. The trophic level of crustaceans has been extensively investigated and, hence, a far higher number of observations (i.e., $\mathrm{EC}_{50}$ ) have been collected compared with the other trophic level: 9 for SWCNT, 37 for MWCNT, and 11 for $\mathrm{C}_{60}$ fullerene.
Fig. 1 Observed $\log \mathrm{EC}_{50}(\mu \mathrm{g} / \mathrm{L})$ modeled $\log \mathrm{EC}_{50}(\mu \mathrm{g} / \mathrm{L})$ values by multiple regression analysis for the group of algae SWCNTMWCNT

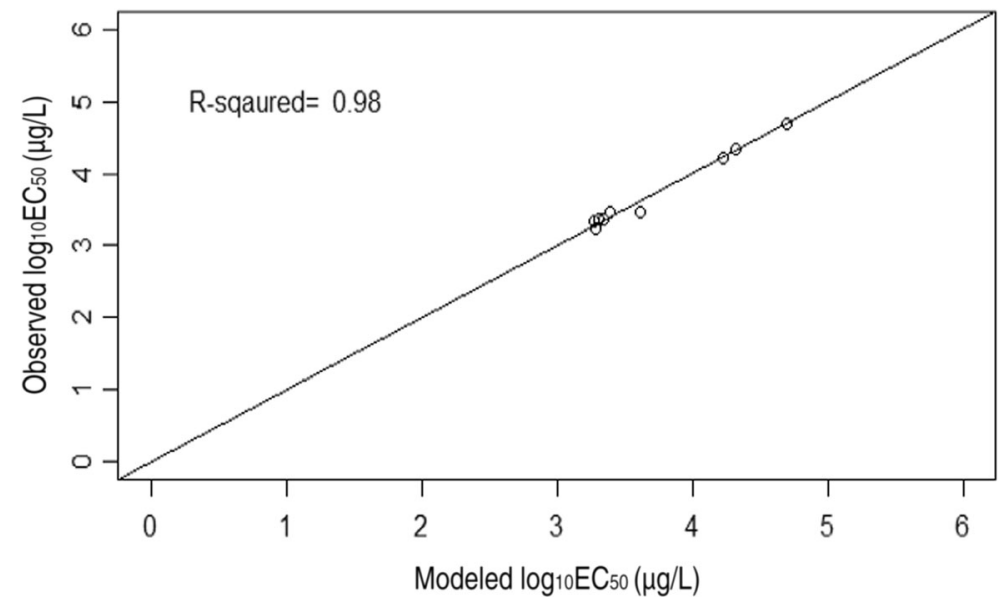


Table 3 Coefficients of MWCNT-algae for multiple linear regression (MRA) and polynomial regression analysis (PRA)

\begin{tabular}{lllllllll}
\hline Algae-MWCNT & $\beta_{0 \text { (intercept) }}$ & $\beta_{1 \text { (diameter) }}$ & $\beta_{1}{ }^{2}$ (diameter) & $\beta_{2 \text { (length) }}$ & $\beta_{2}{ }^{2}$ (length) & $\beta_{3}$ (dispersant) & $\beta_{4}$ (secondary size) & $\beta_{4}{ }^{2}$ (secondary size) \\
\hline MRA & $4.43 \mathrm{E}+00$ & $-2.25 \mathrm{E}-02$ & & $5.03 \mathrm{E}-04$ & & $-2.09 \mathrm{E}+00$ & $-1.59 \mathrm{E}-04$ \\
PRA & $-8.79 \mathrm{e}+03$ & $-8.304 \mathrm{E}+02$ & $9.81 \mathrm{E}+00$ & $2.38 \mathrm{E}+01$ & $-5.62 \mathrm{E}-03$ & $1.55 \mathrm{E}+03$ & $-1.32 \mathrm{E}-02$ & $1.68 \mathrm{E}-05$ \\
\hline
\end{tabular}

In gray, the variables assessed as significant predictors

\section{ANOVA}

The ANOVA analysis has been firstly carried out by grouping the $\mathrm{EC}_{50}$ values by the type of CBNs. Thus, three groups have been identified as SWCNT and MWCNT and $\mathrm{C}_{60}$-fullerene. The difference between the mean $\log \mathrm{EC}_{50}$ of the three groups of nanotubes was significant $(p<0.001)$. The post hoc Tukey test showed that the mean values of the groups SWCNT and MWCNT were significantly different from each other, whereas no significant differences have been observed with $\mathrm{C}_{60}$ fullerene. Thus, the ANOVA's outcomes are different from those observed for the trophic level of algae. For crustaceans, the toxic values reported and applied for SWCNT, MWCNT, and $\mathrm{C}_{60}$-fullerene ranged from 0.05 to $6,1.9$ to 100 , and 0.46 to $7.9 \mathrm{mg} / \mathrm{L}$, respectively. Thus, the range of values reported for SWCNT and $\mathrm{C}_{60}$-fullerene is similar. Based on the ANOVA results, the group of data belong to MWCNT, SWCNT, and $\mathrm{C}_{60^{-}}$ fullerene was then separately investigated.

MWCNT An ANOVA test was undertaken by grouping the $\mathrm{EC}_{50}$ values by the properties of functionalization (yes/

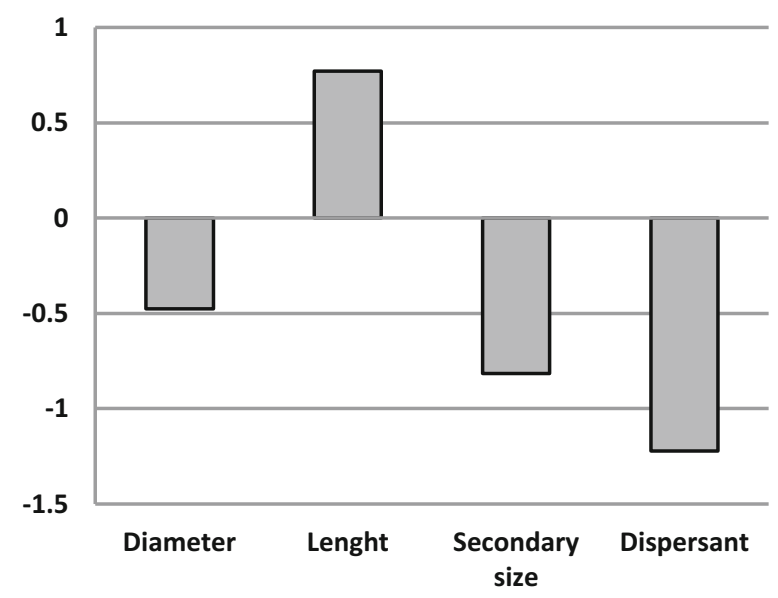

Fig. 2 Standardized $\beta$ linear coefficients for the property of diameter, length, secondary size, dispersant for the group of data algae MWCNT-SWCNT no) and then by use of a treatment to disperse MWCNT in the test solution (yes/no). The functionalization has been assessed as not significant by the ANOVA test $(p=0.4)$. In contrast, the use of treatments (i.e., ozone oxidation) was assessed as significant ( $p=0.03$ ), with lover $\mathrm{EC}_{50}$ values, on average, for the untreated group. Previously, several toxicity studies on functionalized CBNs have been undertaken: hydroxyl group (-OH) (Kennedy et al. 2008), carboxyl group (-COOH) (Kennedy et al. 2008), polyethyleneimene (-PEI) (Petersen et al. 2011), amine $\left(-\mathrm{NH}_{2}\right)$, and alkyl (-C8) (Kennedy et al. 2009) groups. Hydroxyl and carboxyl functionalization could reduce the toxicity of MWCNT on C. dubia (Kennedy et al. 2008), whereas PEI, amine, and alkyl functionalized group could increase the toxicity of MWCNT (Kennedy et al. 2009; Petersen et al. 2011). Different classes of PEI-coated MWCNT (MWCNT-PEI, MWCNT-PEI-Ac, and MWCNT-PEI-Suc) exhibited different toxic values (Petersen et al. 2011). It would be expected that MWCNT-PEI is the most toxic due to it positive surface charge. However, the findings of Petersen et al. (2011) showed that MWCNT-PEI-Ac and MWCNT-PEI-Suc are more toxic than MWCNT-PEI, and therefore, the surface charge must play a less influential role than the size of the polymer coating in the toxicity of MWCNT.

According to previous studies, the physical (i.e., sonication, stirring) and chemical (e.g., ozone oxidation) solution treatments can have a pronounced effect on the stability and aggregation of CBNs and thereby impact on the experimental toxicity on freshwater organisms. For example, sonication was found to be more effective at dispersing MWCNTs into stable suspensions and thus result in higher stable concentration compared to magnetic stirring (Kennedy et al. 2009). Li and Huang (2011) showed that ultrasound treatment (US) of MWCNT exerted the greatest toxicity to $C$. dubia compared to the untreated and ozonetreated MWCNT, which exhibited a $\mathrm{LC}_{50}$ of one order of magnitude greater than that of US-MWCNT. These results indicate that US treatment could drastically increase the toxicity of MWCNT. This trend was observed for both a 24-h acute test and with a 3-brood reproduction assay. In 
fact, the significantly higher mean $\mathrm{EC}_{50}$ value observed in the present analysis for the group where a dispersion treatment was used is due to the three cases where the treatment was ozone oxidation (each case being from the same study [Li and Huang 2011]). While MWCNTs of different diameters were used for each case, the $\mathrm{EC}_{50}$ was the same $(100 \mathrm{mg} / \mathrm{L})$ for the three of them. This value is two to three orders of magnitude higher than all the other values included in the comparison.

SWCNT A lack of physicochemical information reported in literature prevented the performance of the ANOVA test.

$C_{60}$-fullerene The ANOVA test has been carried out by grouping the $\mathrm{EC}_{50}$ values by the use of dispersant (yes/no), with the use of dispersant (here tetrahydrofuran, THF) found to be significant $(p<0.001)$. The use of THF has already been widely discussed in the literature (Handy et al. 2012; Lovern and Klaper 2006, Gao et al. 2012), and several concerns have been raised in the field of ecotoxicity since THF might affect the outcomes of the toxicity test. In our survey, even though few observations were collected, the lower $\mathrm{EC}_{50}$ values are referred to the ecotoxicity tests where THF was applied (Table S8). Gao et al. (2012) tested several types of surfactants, both on Ceriodaphnia dubia and Pseudokirchnerella subcapitata. The study revealed that the biological effects of tested surfactants (THF, PVP, GA, Triton-X, Na-cholate, SDS) varied with the concentration and type of tested model organism. The lower $\mathrm{EC}_{50}$ values were found for THF on both the organisms tested. However, further investigations are required to assess the contribution of THF to the overall toxicity of the solution. Lovern and Klaper (2006) compared the use of THF in solution with nano- $\mathrm{TiO}_{2}$ with a filtered solution of nano- $\mathrm{TiO}_{2}$ by testing the solutions on D. magna with endpoint of mortality. The study revealed that no statistical difference was found among the two types of solution and, furthermore, that the THF was not the cause of mortality on D. magna. Henry et al. (2007) exposed larval zebrafish to THF-C $\mathrm{C}_{60}$, THF-water, and $\mathrm{C}_{60}$-water and investigated the changes in survival and gene expression. Survival was reduced in THF- $_{60}$ and THF-water but not in $\mathrm{C}_{60}$ water, and the greatest differences in gene expression were observed in fish exposed to THF-C 60 . The gas chromatography-mass spectrometry investigation on the solution tested revealed that THF was not detected but revealed the presence of THF oxidation products, such as gamma-butyrolactone and tetrahydro-2-furanol. Thus, the research performed by the authors indicated that the effects in THF treatments can result from gamma-butyrolactone toxicity, and more, it linked the toxic effect to THF degradation product rather than to $\mathrm{C}_{60}$.

\section{Regression analysis}

Based on the outcomes of the ANOVA, regression analysis investigation has been carried out without merging the data collected for MWCNT-SWCNT and fullerene.

MWCNT The statistical analysis was negatively affected by the incompleteness of available data. In many studies, not all of the physicochemical characteristics of interest were reported. Due to the excessive number of missing data, it was therefore not possible to evaluate the effect of all the variables on the toxicity using the whole data set. To overcome this problem (at least partially), two groups of analyses were conducted. The first was performed on data from all studies on MWCNTs that were collected and included as predictors: diameter, secondary size, use of dispersant (here, natural organic matter), and duration of the exposure. The second was carried out on the subset of those data that included length as an additional predictor.

Regarding the first group of analyses, the regression coefficients $(\beta)$ of the multiple linear regressions are shown in Table 4. All the variables, with the exception of diameter, have been assessed as significant predictors; the most influential independent properties on the toxic values are reported in (Fig. 3). The predicted log $\mathrm{EC}_{50}$ values correlate fairly well $\left(p=0.028 ; R^{2}=0.65\right)$

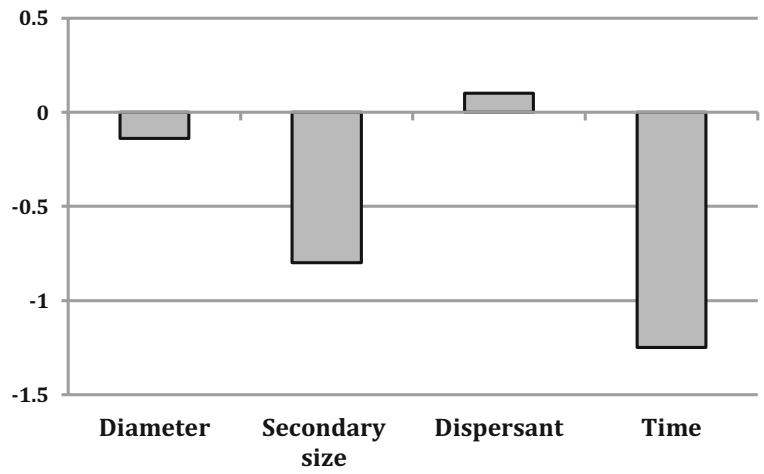

Fig. 3 Standardized $\beta$ linear coefficients for the property of diameter, secondary size, dispersant and time for the group of data crustaceans- MWCNT. 
Table 4 Coefficients of MWCNT-crustaceans for multiple linear regression (MRA) and polynomial regression analysis (PRA)

\begin{tabular}{lllllllll}
\hline Crustaceans-MWCNT & $\beta_{0 \text { (intercept) }}$ & $\beta^{1}{ }_{\text {(diameter) }}$ & $\beta_{1}{ }^{2}{ }_{\text {(diameter) }}$ & $\beta_{3 \text { (time) }}$ & $\beta_{3}{ }^{2}$ (time) & $\beta_{4}$ (secondary size) & $\beta_{4}{ }^{2}$ (secondary size) & $\beta_{5}$ (dispersant) \\
\hline MRA & $5.67+00$ & $-5.89 \mathrm{E}-03$ & & $-2.19 \mathrm{E}-02$ & $-9.66 \mathrm{E}-05$ & $1.24 \mathrm{E}-01$ \\
PRA & $6.48 \mathrm{E}+00$ & $-3.13 \mathrm{E}-02$ & $3.51 \mathrm{E}-04$ & $-1.84 \mathrm{E}-02$ & $\mathrm{NA}$ & $-5.45 \mathrm{E}-00$ & $3.55 \mathrm{E}-04$ & $-2.95 \mathrm{E}-01^{1}$ \\
\hline
\end{tabular}

The variable of length is excluded. In gray, the variables assessed as significant predictors

$N A$ not analyzed value due to collinearity or singularities

with the measured values of the MWCNT reported for the trophic level of crustaceans.

The polynomial regression coefficients $\beta$ and $\beta^{2}$ have been estimated for all the properties, with exception of the time. However, with the exception of secondary size, the $\beta^{2}$ coefficients are not significant. This indicates that a monotonic relationship exists between the dependent variable and the independent variables. Notably, the first-order coefficient of these last properties is negative, whereas the quadratic coefficient is positive. This suggests that the relationship between secondary size and the $\mathrm{EC}_{50}$ is not monotonic and that the $\mathrm{EC}_{50}$ decreases (i.e., toxicity increases) as secondary size increases up to a point where the toxicity is the highest. At this point, the relationship changes direction and the $\mathrm{EC}_{50}$ decreases as secondary size increases further.

Overall, the PRA model is highly significant ( $p$ value $<0.001)$ and the predicted $\log$ of the $\mathrm{EC}_{50}$ values correlate well $\left(R^{2}=0.86\right)$ with the measured values of the MWCNT reported for the trophic level of crustaceans. The data points lying close to the regression line reveal the good fit of the predictive model (Fig. 4).

A second subgroup of data for crustaceans-MWCNT has been established by including the physicochemical property of length and excluding all the cases for which this property was not reported. The polynomial linear regression coefficients $(\beta)$ of the MRA are shown in Table 5. Diameter and time of exposure are assessed as significant predictors and the secondary size assessed as not significant predictor. Coefficients $(\beta)$ for the variable of length are not calculated due to collinearity. The multiple linear regression analysis results in a $p$ value $<0.001$ and $R^{2}=0.97$.

The MRA can be accepted ( $p$ values $<0.001$ ) and the predicted $\log$ of the $\mathrm{EC}_{50}$ values correlates well $\left(R^{2}=0.97\right)$ with the measured values of the MWCNT reported for the trophic level of crustaceans. The data points (Fig. 5) of the lying close to the regression line revealed the good fit of the predictive model (Eq. (1)).

A similar trend is observable within the PRA. However, diameter was here assessed as being a nonsignificant predictor. Only time was identified as being a significant predictor. The polynomial regression analysis has a $p$ value of $<0.001$; the predicted log of the $\mathrm{EC}_{50}$ values correlates well $\left(R^{2}=0.97\right)$ with the measured values of the MWCNT reported for the trophic level of crustaceans (Fig. 4).

In summary, the regression analysis suggests that secondary size has a nonmonotonic effect on the toxicity of MWCNT on crustaceans. No other property of the CBN was found to have a significant effect on toxicity. As expected, the $\mathrm{EC}_{50}$ significantly decreases as the exposure time increases.

SWCNT The variables of diameter, length, secondary size, and use of dispersant (here, NOM) have been collected as independent variables for the MRA and PRA. However, only four observations were available for SWCNT for the regression analysis. In this case of study, the number of the observations is equal to the number of the variables to investigate, and thus, the investigation by the regression analysis was impossible to perform.

$C_{60}$ fullerene The investigation by regression analysis was impossible to perform, mainly due the lack of information regarding the physicochemical properties.

Trophic level of fish

The physicochemical information together with the ecotoxicity data for the trophic level of fish for MWCNT, SWNCT, and fullerene is reported in Tables S3-S9.

SWCNT To date, only two toxicity studies have been performed for SWCNT (Table S3). Toxicity has been assessed with two species, Danio rerio and Oryzias latipes, with the endpoint of hatching delay and mortality, respectively. The studies identified the toxic values of LOEC (120 mg/L) (Cheng et al. 2007) and $\mathrm{LC}_{50}(96 \mathrm{~h})$ of $100 \mathrm{mg} / \mathrm{L}$ (Sohn et al. 2015). Notably, the latter one corresponds to the highest nominal concentration tested. 
A

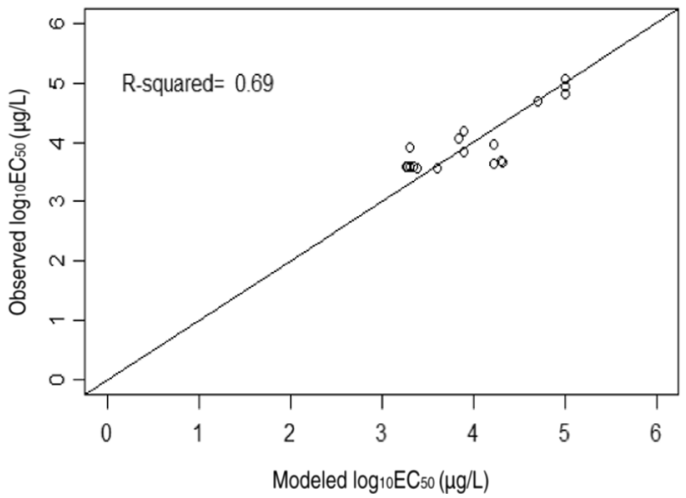

B

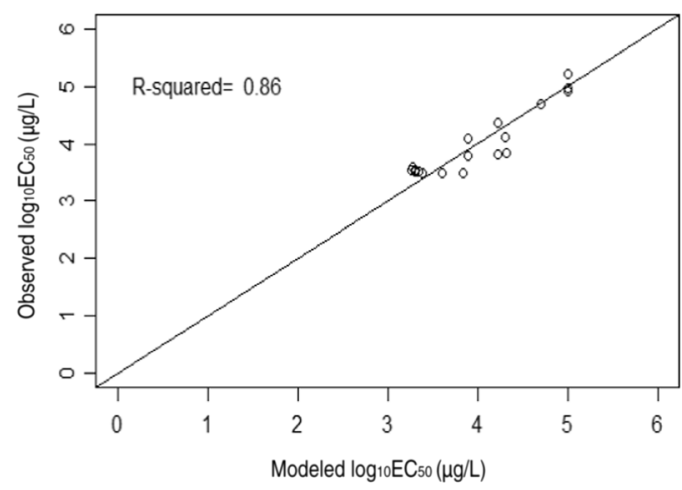

Fig. 4 Observed versus modeled $\log _{10} \mathrm{EC} 50(\mu \mathrm{g} / \mathrm{L})$ values for the group crustaceans-MWCNT (length not included). a multiple linear regression $\mathbf{b}$ polynomial regression

However, the scarce number of observations (i.e., two studies) has prevented the formulation of the correlation matrix as well as the undertaking of the ANOVA or regression analysis.

$M W C N T$ The ecotoxicity data on fish and the physiochemical properties of MWNCT are reported in Table S6. Only three observations (i.e., LOEC and $\mathrm{EC}_{50}$ ) have been found in the literature (Asharani et al. 2008). The toxicity of MWCNT on fish has been assessed in relation to the species Danio rerio with the endpoints of mortality and phenotypic defects. Here, the scarcity of toxicity studies is the main obstacle. Cheng and Cheng (2012) assessed the toxicity of MWCNT on zebrafish by several endpoints, namely subcellular morphology change and failure of epiboly initiation in embryos. MWCNTs were sonicated in a nitric acid solution for 24 and $48 \mathrm{~h}$, following which a different size of length was observed. MWCNTs prepared with the longer sonication time induced severe developmental toxicological effects, whereas the shorter sonication time did not induce any toxicity.
Overall, Cheng and Cheng (2012) suggested that length can play an important role in the in vivo toxicity of MWCNT.

Fullerene A summary of the physiochemical properties of fullerene and its toxic effects on fish is reported in Table S9. Five fish species, juvenile carp (Carassius aratus), zebrafish (Danio rerio), largemouth bass (Micropterus salmoides), Japanese rice fish (Oryzias latipes), and fathead minnow (Pimephales promelas), were used as testing organisms. A number of endpoints were reported, namely mortality, growth inhibition by body weight, growth inhibition by body length, sublethal morphological and developmental effect, and lipid peroxidation in the brain.

A high variability in toxic values is observable; the toxic values range from $\mathrm{LOEC}=0.2 \mathrm{mg} / \mathrm{L}$ (Zhu et al. 2009) to $\mathrm{LC}_{50}=4 \mathrm{mg} / \mathrm{L}$ (Usenko et al. 2007). Few studies were found in the literature and, hence, few observations (i.e., $4 \mathrm{LC}_{50} \mathrm{~s}$ ), with a scarce characterization (only the secondary size is reported), were

Table 5 Coefficients of MWCNT-crustaceans for multiple regression and polynomial regression analysis

\begin{tabular}{llllllllll}
\hline Crustaceans-MWCNT & $\beta_{0 \text { (intercept) }}$ & $\beta_{\text {(diameter) }}^{1}$ & $\beta_{1}{ }^{2}$ (diameter) & $\beta_{2 \text { (length) }}$ & $\beta_{2}{ }^{2}$ (length) & $\beta_{3}$ (time) & $\beta_{3}{ }^{2}$ (time) & $\begin{array}{l}\beta_{4} \\
\text { (secondary size) }\end{array}$ & $\begin{array}{l}\beta_{4}{ }^{2} \\
\text { (secondary size) }\end{array}$ \\
\hline $\begin{array}{c}\text { Multiple } \\
\text { regression analysis }\end{array}$ & 10.47 & -0.28 & & NA & & -0.008 & & 0.0010 \\
$\begin{array}{c}\text { Polynomial } \\
\text { regression analysis }\end{array}$ & $8.56 \mathrm{E}+00$ & $-1.31 \mathrm{E}-01$ & $\mathrm{NA}$ & $\mathrm{NA}$ & $\mathrm{NA}$ & $-1.05 \mathrm{E}-02$ & $\mathrm{NA}$ & $-4.44 \mathrm{E}-03$ & $4.64 \mathrm{E}-06$ \\
\hline
\end{tabular}

In gray, the variables assessed as significant predictors

$N . A$ the coefficient is not estimable (i.e., due to collinearity or due to a low number of observation) 
A

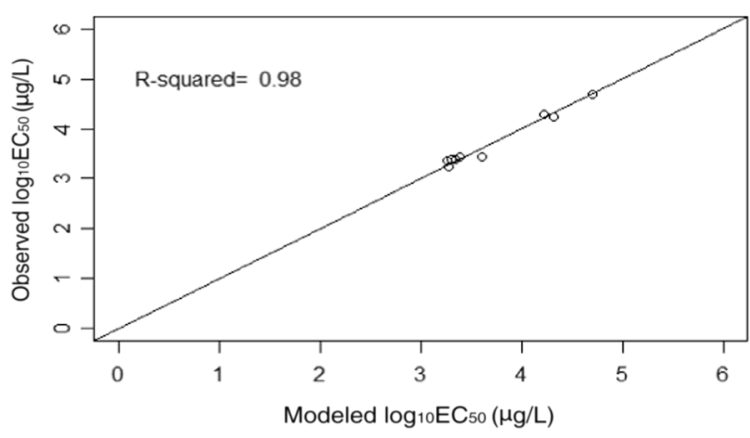

B

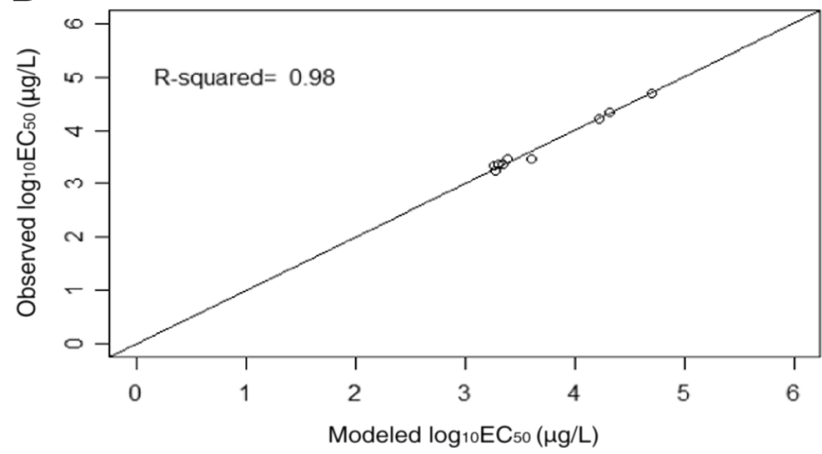

Fig. 5 Observed versus modeled $\log _{10} \mathrm{EC} 50(\mu \mathrm{g} / \mathrm{L})$ values for the group crustaceans-MWCNT (length included) with MRA (a) and PRA (b)

collected. Consequently, the investigation on fullerene for the trophic level of fish was impossible to perform. Indeed, the high variability reported in and the low characterization of the ENM literature could, by itself, affect the outcomes.

\section{Conclusion}

Based on the use of established statistical approaches, this article investigated the relationship between physicochemical properties of CBNs and observed toxic effects. However, the investigation was restricted by due to the paucity of relevant, available data (i.e., mean $\mathrm{EC}_{50}$ values for ENM toxicity for each trophic levels), and by the low variability in available data. Indeed, it can be observed that the $\mathrm{EC}_{50}$ values collected were often obtained from two or three studies only. Therefore, the investigation (i.e., ANOVA) performed here might identify rather the intrinsical difference among the few studies than a significant difference between the properties of the various groups (i.e., the lengths of the $\mathrm{CBNs}$ ).

The importance of the number of data points collected (at each trophic level) and their quality in terms of source variability is confirmed by the regression analysis here performed. Indeed, the regression analysis failed several time, while the coefficient predictors were not evaluated in certain instances due to the presence of singularities. Despite that our findings are strongly influenced by the scarcity of data (e.g., in several cases the PRA failed due to case of singularities), the PRA investigations here still can elucidate whether (1) a parameter is significant both in MRA and PRA (and thus confirm the linear and monotonic relationship), or (2) if the statistical significance of parameters changes. The regression analysis conducted on MWCTN-Crustacean showed that similar outcomes were obtained for the MRA and PRA. Indeed, time and secondary size are significant parameters for both MRA and PRA, whereas diameter and dispersant use are not significant either case. For the variable of diameter, the second-order coefficient from the PRA was found to be significant, suggesting that diameter has a nonmonotonic effect on the toxicity of MWCNT. A similar comparison can be done for the investigation on crustacean toxicity from MWCNT for which the outcomes of the MRA and PRA are the same. Furthermore, the parameter of time was revealed to be significant for both the two models. Diameter was found to be significant with MRA but not with PRA. In the latter case, the coefficient at second grade was not evaluable due to the occurrence of singularities. Our results indicate that the PRA was strongly affected by the low number of data collected.

Although physical properties of CBNs are the important properties to define toxicity, some studies have not appropriately reported their physical properties. Due to the scarcity of information reported in the literature, the issue of impurities in the synthesized nanomaterials has not been investigated - although, like in the case of metal catalyst residues from the SWCNT synthesis, they are proposed to be a possible cause of toxicity in various publications (Gao et al. 2012; Sohn et al. 2015). The characterization of CBNs should be performed thoroughly to improve the quality of toxicity data. The correlation analysis should be performed further in the future, when the availability and quality of data are improved. Expressly designed experiments would be the best tool to clarify the effect of ENMs properties on their toxicity. This holds especially if the aim is to clarify the interactive effects of different properties. However, given the numbers of 
groups of ENMs, different potentially relevant properties, and possible biological targets, systematic investigation based on the application of statistical approaches to published studies could have a major role. The success of this approach in the future would require that the ENMs and the experimental conditions used in the toxicity studies are fully characterized.

Acknowledgements The research leading to these results has received funding from the European Union Seventh Framework Programme under grant agreement no. 310184 (CARINHYPH project).

\section{Compliance with ethical standards}

Conflict of interest The authors declare that they have no conflict of interest.

Human and animal studies This article does not contain any studies with human or animal subjects performed by any of the authors.

\section{References}

Alloy MM, Roberts AP (2011) Effects of suspended multi-walled carbon nanotubes on daphnid growth and reproduction. Ecotoxicol Environ Saf 74(7):1839-1843

Arndt DA, Moua M, Chen J, Klaper RD (2013) Core structure and surface functionalization of carbon nanomaterials alter impacts to daphnid mortality, reproduction, and growth: acute assays do not predict chronic exposure impacts. Environ Sci Technol 47(16):9444-9452

Arts JH-E, Hadi M, Irfan MF, Keene AM, Kreiling R et al (2015) A decision-making frameworj for the gruoping and testing of nanomaterials (DF4nanoGruoping). Regul Toxicol Pharmacol 71(2):s1-s27

Aschberger K, Johnston HJ, Stone V, Aitken RJ, Tran CL et al (2011a) Review of fullerene toxicity and exposure - appraisal of a humna health risk assessment, based on open literature. Regul Toxicol Pharmacol 58:455-473

Aschberger K, Micheletti C, Sokull-Kluttgen B, Christensen FM (2011b) Analysis of currently available data for characterising the risk of engineered nanomaterials to the environment and human health - lessons learned from four case studies. Environ Int 37:1143-1156

Asharani PV, Serina NGB, Nurmawati MH, Wu YL, Gong Z, Valiyveettil S (2008) Impact of multi-walled carbon nanotubes on aquatic species. J Nanosci Nanotechnol 8(7):36033609

Cheng J, Cheng SH (2012) Influence of carbon nanotube length on toxicity to zebrafish embryos. Int J Nanomedicine 7:3731-3739

Cheng J, Flahaut E, Cheng SH (2007) Effect on carbon nanotubes on developing zebrafish (Danio rerio) embryos. Environ Toxicol Chem 26:708-716

Coll C, Notter D, Gottschalk F, Sun TY, Som C, Nowack B (2016) Probabilistic environmental risk assessment of five nanomaterials (nano-TiO2, nano-Ag, nano-ZnO, CNT, fullerenes). Nanotoxicology 10:36-444

ECHA (2013) Gruoping of substance and read-across approach an illustraive example

Edgington AJ, Roberts AP, Taylor LM, Alloy MM, Reppert J, Rao AM et al (2010) The influence of natural organic matter on the toxicity of multiwalled carbon nanotubes. Environ Toxicol Chem 29(11):2511-2518

Gao J, Llaneza V, Youn S, Silvera-Batista CA, Ziegler KJ, Bonzongo JC (2012) Aqueous suspension methods of carbon-based nanomaterials and biological effects on model aquatic organisms. Environ Toxicol Chem 31:210-214

Gottschalk F, Sonderer T, Scholz RW, Nowack B (2009) Modeled environmental concentrations of engineered nanomaterials ( $\mathrm{TiO} 2, \mathrm{ZnO}, \mathrm{Ag}, \mathrm{CNT}$, fullerenes) for different regions. Environ Sci Technol 43:9216-9222

Gottschalk F, Sun TY, Nowack B (2013) Environmental concentrations of engineered nanomaterials: review of modeling and analytical studies. Environ Pollut 181:287-300

Guarch CP, López DR, Salse JT, Linares JG, Suàrez MB, de Lapuente PJ (2014) Basis for the toxicological evaluation of engineered nanomaterials. Rev Toxicol 31(1):9-22

Handy DR, Owen R, Valsami-Jones E (2008) The ecotoxicology of nanopaeticles and nanomaterails: current status, knowledge gaps, challenges, and future needs. Ecotoxicology 17:315-325

Handy DR, Cornelis G, Fernandes T, Tsyusko O, Decho A et al (2012) Ecotoxicity test methods for engineered nanomaterials practicla experiences and recommendations from the bench. Environ Toxicol Chem 31(1):15-31

Henry TB, Menn FM, Fleming JT, Wiligus J, Compton RN, Sayler GS (2007) Attributing effects of aqueous C60 nanoaggragtes to tetrahydrofuran decomposition products in larval zebrafish by assessment of gene expression. Environ Health Perspect 115(7):1059-1065

Hischier R (2014) Framework for LCI modelling of releases of manufactured nanomaterials along their life cycle. Int J LCA 19:941-943

Hischier R, Walser T (2012) Life cycle assessment of engineered nanomaterials: state of the art and strategies to overcome existing gaps. Sci Total Environ 425:271-282

ISO (2006) Environmental management - life cycle assessment life cycle impact assessment (ISO 14044). ISO, Geneva

Jackson P, Jacobsen NR, Baun A et al (2013) Bioaccumulation and ecotoxicity of carbon nanotubes. Chem Cent J 7:154

Kapler R, Crago J, Barr J, Arndt D, Setyowati K, Chen J (2009) Toxicity biomarker expression in daphnids exposed to manufactured nanoparticles; changes in toxicity with functionalization. Environ Poll 157:1152-1156

Kennedy AJ, Hull MS, Steevens JA, Dontsova KM, Chappell MA, Gunter JC, Weiss CA (2008) Factors influencing the partitioning and toxicity of nanotubes in the aquatic environment. Environ Toxicol Chem 27(9):1932-1941

Kennedy AJ, Gunter JC, Chappell MA, Goss JD, Hull MS, Kirgan RA, Steevens JA (2009) Influence of nanotube preparation in aquatic bioassays. Environ Toxicol Chem 28(9):1930-1938

Kim KT, Klaine SJ, Lin S, Ke PC, Kim SD (2010) Acute toxicity of a mixture of copper and single-walled carbon nanotubes to Daphnia magna. Environ Toxicol Chem 29(1):122-126 
Li M, Huang CP (2011) The responses of Ceriodaphnia dubia toward multi-walled carbon nanotubes: effect of physicalchemical treatment. Carbon 49(5):1672-1679

Liu Y, Zhao Y, Sun B, Chen C (2013) Understanding the toxicity of carbon nanotubes. Acc Chem Res 46:702-713

Long Z, Ji J, Yang K, Lin D, Wu F (2012) Systematic and quantitative investigation of the mechanism of carbon nanotubes toxicity toward algae. Environ Sci Technol 46(15):8458-8466

Lovern S, Klaper R (2006) Daphnia magna Mortality when exposed to titanium dioxide and fullerene (C60) nanoparticles. Environ Toxicol Chem 25(4):1132-1137

Luo J (2007) Toxicity and bioaccumulation of nanomaterial inaquatic species. JUS SJWP 2:1-16

Mueller NC, Nowack B (2008) Exposure modeling of engineered nanoparticles in the environment. Environ Sci Technol 42: $4447-4453$

Muzi L, Tardani F, La Mesa C, Bonincontro A, Bianco A, Risuleo $\mathrm{G}$ (2016) Interactios and effects of BSA-functionalized single-walled carbon nanotubes on different cell lines. Nanotechnology 1-10

Oomen AG, Peter MJ, Fernandes TF, Hund-Rinke K, Boraschi D et al (2014) Concern-driven integrated approaches to nanomaterial testing and assessment - report of the NanoSafety cluster working group 10. Nanotoxicology 8:334-348

Park EJ, Lee GH, 'Han BS , Lee BS, Lee S, Cho MH, Kim JH, Kim DW (2015) Toxic response of graphene nanoplatelets in vivo and in vitro. Arch Toxicol 89:1557-1568.

Pereira MM, Mouton L, Yéprémian C, Couté A, Lo J et al (2014) Ecotoxicological effects of carbon nanotubes and cellulose nanofibers in Chlorella vulgaris. J Nanobiotechnol 12(15):1-13

Petersen EJ, Pinto RA, Mai DJ, Landrum PF, Weber WJ (2011) Influence of Polyethyleneimine Graftings of multi-walled carbon nanotubes on their accumulation and elimination by and toxicity to Daphnia magna. Environ Sci Technol 45(3):1133-1138

Rivera-GP, Jimenez de Aberasturi D, Wulf V, Pelaz B, Del Pino P et al (2012) The challenge torelate the physicochemial properties of colloidal nanoparticles to their cytotoxicity. Acc Chem Res 46:743-749

Roberts AP, Mount AS, Seda B, Souther J, Qiao R et al (2007) In vivo Biomodification of lipid-coated carbon nanotubes by Daphnia magna. Environ Sci Technol 41(8):3025-3029

Rosenbaum R, Bachmann T, Gold L, Huijbregts MJ, Jolliet O et al (2008) USEtox - the UNEP-SETAC toxicity model: recommended characterisation factors for human toxicity and freshwater ecotoxicity in life cycle impact assessment. Int J LCA 13(7):532-546
Rossi M, Cubadda F, Dini L, Terranova ML, Aurele F, Sorbo A, Passeri D (2014) Scientific basis of nanotechnolgoy, implication for the food sector and future trend. Trend Food Technol 40(2):127-148

Schwab F, Bucheli TD, Lukhele LP, Magrez A, Nowack B, Sigg L, Knauer K (2011) Are carbon nanotube effects on green algae caused by shading and agglomeration? Environ Sci Technol 45(14):6136-6144

Seda BC, Ke P-C, Mount AS, Klaine SJ (2012) Toxicity of aqueous C70-Gallic acid suspesnion in Daphnia magna. Environ Tox Chem 31:215-220

Sohn EK, Chung YS, Johari SA, Kim TG, Kim JK, Lee JH, Lee YH, Kang SW, Yu IJ (2015) Acute toxicity comparison of single-walled carbon nanotubes in various freshwater organisms. Biomed Res Int 2015:323090

Subramanian V, Semenzin E, Hristozov D, Zondervan-van den Beuken E, Linkov I, Marcomini A (2015) Review of decision analytic tools for sustainable nanotechnology. Environ Syst Decis 35:29-41

Tao X, Fortner JD, Zhang B, He Y, Chen Y, Hughes JB (2009) Effects of aqueous stable fullerene nanocrystals (nC60) on Daphnia magna: evaluation of sub-lethal reproductive responses and accumulation. Chemosphere 77(11):1482-1487

Usenko CY, Harper SL, Tanguay RL (2007) In vivo evaluation of carbon fullerene toxicity using embryonic zebrafish. Carbon NY 45(9):1891-1898

Usenko CY, Harper SL, Tanguay RL (2008) Fullerene C60 exposure elicits an oxidative stress response in embryonic zebrafish. Toxicol Appl Pharmacol 229(1):44-55

Valverde LJ, Linkov I (2011) Nanotechnology: risk assessment and risk management perspective. Nanotechnol Law Bus 8:1-23

Youn S, Wang R, Gao J, Hovespyan A, Ziegler KJ, Bonzongo JC, Bitton G (2012) Mitigation of the impact of single-walled carbon nanotubes on a freshwater green algae: Pseudokirchneriella subcapitata. Nanotoxicology 6(2):161-172

Yan XM, Zha JM, Shi BY et al (2010) In vivo toxicity of nano-C60 aggregates complex with atrazine to aquatic organisms. Chinese Sci Bull 55(4-5):339-345

Zhu S, Oberdorster E, Haasch ML (2006) Toxicity of an engineered nanoparticle (fullerene, C60) in two aquatic species, Daphnia and fathead minnow. Mar Environ Res 62(Suppl):S5-S9

Zhu X, Zhu L, Chen Y, Tian S (2009) Acute toxicities of six manufactured nanomaterial suspensions to Daphnia magna. J Nanopart Res 11(1):67-75 